Classification

Physics Abstracts

$42.40-42.65$

\title{
Influence of the density of photorefractive centers on volume holographic capacity storage
}

\author{
Phannara Aing, Clara Alves, Gilles Pauliat and Gérald Roosen \\ Institut d'Optique, Unité de Recherche Associée 14 au Centre National de la Recherche \\ Scientifique, Bât. 503, Centre Scientifique, B.P. 147. 91403 Orsay Cedex, France
}

(Received 14 March 1994, revised 16 August 1994, accepted 10 Octoher 1994)

\begin{abstract}
We discuss the influence of the limited available number of photorefractive centers on the holographic storage capacity of phase holograms in photorefractive materials. We compare this limitation with the other sources of noise that also limit the holographic storage capacity of photorefractive materials. As an example, we apply our formulae to gray level images stored in a $\mathrm{BaTiO}_{3}$ photorefractive sample.
\end{abstract}

\section{Introduction.}

Various schemes for volume holographic storage in photorefractive materials have already been proposed and demonstrated [1-9]. This photorefractive effect is now a very well known phenomenon [10]. It arises from the existence of electric charge centers (growth defects or doping centers) distributed at random in the crystal volume. These centers are partly occupied by electric charges, electrons or holes. Under illumination, these electrons (or holes) are excited in the conduction (or valence) band. Then, they migrate in the crystal until they recombine in another empty center. This process results in a depletion of electrons in the regions of largest illumination and accumulation of electrons in the others. For instance a spatially periodic illumination, such as the one created by the interference of two light beams, induces a charge grating and thus an electric field grating of same period. An index grating results from the Pockels effect. The photorefractive effect thus allows the real time recording of holograms. Because of the low dark conductivity of photorefractive crystals, these holograms are stable in the dark. The reconstruction of the image beam by a reference beam releases the electric charges and thus erases the stored information. However various techniques, such as fixing processes [11-17] non destructive readout methods [18, 19] or refreshing procedures [20-23], may be employed to prevent this loss of information so that photorefractive crystals may be used as dynamic (write/erase) memories with a long term storage.

Most photorefractive materials are available in high optical quality and large sizes. Thus, taking advantage of the angular or wavelength selectivity (Bragg effect) of thick crystals, many 
holograms can be superposed in the same volume [24]. They form a stack of images. In order to further increase the storage capacity, several stacks may be juxtaposed side by side in the same crystal volume.

In principle, one may think that very large capacity memories can be constructed using this photorefractive effect. This was the aim of many experiments performed up to now. These demonstrations were supported by the work of van Heerden [25] who determined the maximum theoretical capacity of optical volume data storage to be about $\mathrm{Vol} / \lambda^{3}$, with Vol the crystal volume and $\lambda$ the optical wavelength in the material. These data being either binary, bits, or multilevel data if the signal to noise ratio is large enough. As an example, for a crystal of volume $5 \times 5 \times 5 \mathrm{~mm}^{3}$, a wavelength $\lambda=220 \mathrm{~nm}$, the theoretical limit gives a capacity $c \approx 10^{13}$ data. However, the maximum number of stored images in a single photorefractive crystal was 5000 video images with $320 \times 220$ pixels each in a $3 \mathrm{~cm}^{3}$ crystal [6]. In that case, the capacity was about $10^{8}$ data (gray level pixels). This value seems to be about the maximum that can be holographically stored in a single stack of a photorefractive crystal.

One of the reasons for this limitation, that we will discuss in detail below, is that the calculations performed by van Heerden cannot be applied to usual holographic storage. After this discussion, we will derive the valid expressions for maximum storage capacity of holographic materials. We will briefly derive an estimate of maximum storage capacity of color center materials and we will describe in more details the storage capacity of photorefractive materials. Then, because of the finite usable optical power, we will demonstrate that a trade off must be found between the capacity and the output data rate. Before concluding, as an example, we will apply all these results to determine the storage capabilities and limitations of one of the most commonly used photorefractive crystals.

\section{Some comments about the ultimate capacity of volume storage.}

van Heerden estimated the optical volume data storage capacity as follow. First, he calculated the minimum size of a storage cell. This size is limited by diffraction only so that its volume is about $v \approx \lambda^{3}$. Thus in the total crystal volume Vol, the total amount of information cells is $c \approx \mathrm{Vol} / \lambda^{3}$. Furthermore, if in each cell a data can be stored and retrieved with a large enough amplitude signal to noise ratio SNR, according to Shannon definition, the total amount of stored bits is :

$$
c \approx \frac{\mathrm{Vol}}{\lambda^{3}} \log _{2}(\mathrm{SNR})
$$

He took the example of color center crystals in which holograms are stored by bleaching these centers. If the density of color centers is $N_{\mathrm{cc}}$, he estimated the total amount of bleached color centers per stored hologram to be $N_{\mathrm{cc}} \mathrm{Vol} / \mathrm{V} \mathrm{c}$. However the main source of noise is due to the random distribution of color centers. The noise amplitude is thus proportional to $\sqrt{N_{\mathrm{cc}} \text { Vol. In }}$ that case, the amplitude signal to noise ratio is :

$$
\mathrm{SNR} \approx \frac{\sqrt{N_{\mathrm{cc}} \mathrm{Vol}}}{\sqrt{c}}=\sqrt{N_{\mathrm{cc}} \lambda^{3}} .
$$

Combining equation (1) with equation (2) leads to very large capacities. We will now show in the next paragraph that this theoretical capacity can never be reached with angular holographic multiplexing. The reason is that van Heerden assumed that all data are recorded in a single exposition. This recording procedure leads to very large signal to noise ratios. We will explain why the recording of several holograms in the same material volume leads to much poorer 
signal to noise ratios. For elementary cells with ultimate volume $v \approx \lambda^{3}$, the signal to noise ratios are much lower than unity so that formula (1) is no more valid.

\section{Holographic storage.}

To independently retrieve the images stored in a single stack, the angular or wavelength Bragg selectivity is used. For instance in the reference beam angular multiplexing, the crystal orientation and the image beam incident angle are constant. The address of each image is represented by the incident angle of the reference beam which is changed for each image recording. The images are retrieved by reading out with the reference beam set at the same incident angle that was used for recording. If the spacings between the angles are large enough, the images are retrieved with negligible crosstalk. In wavelength multiplexing, the optical wavelength of the recording beams represents the address of the image. Different other encoding techniques have been proposed and demonstrated such as the "rotating crystal multiplexing ", the « reference beam phase encoding » or the « electric field encoding ». All these techniques use the Bragg selectivity and it has been demonstrated that their image capacities are limited by the same geometrical constraints [26]. All of them also suffer from the same limitation arising from noise due to the random distribution of electric charges. For sake of simplicity we will discuss this limitation taking the example of the reference beam angular multiplexing. However, similar discussions, leading to similar results, could be developed for the other multiplexing schemes.

3.1 Modulation Ratio of ANGUlarly multipleXed holograms. - Experimental scheme for angular multiplexing of $N$ images with $\sqrt{M} \sqrt{M}$ pixels each may be represented as shown in figure 1. The reference beam is assumed to be a plane wave and the image beam is composed of uniform plane waves. Their intensities represent the pixel values. The wave vector of the reference beam for the recording of the $n$th image is $k_{\text {ref }}^{n}$. Similarly we note $\mathbf{k}_{m}^{n}$ the wave vector of the $m$ th pixel beam for the recording of the same $n$th image. With similar notations, the amplitudes of the beams are $A_{\text {ref }}^{n}$ and $A_{m}^{n}$. The pixel value is encoded either on the amplitude $A_{m}^{n}$ or on the phase of that beam while the reference beam amplitude $A_{\text {ref }}^{n}$ is the same whatever the recorded image. For each exposure, we thus record two kinds of gratings : first the gratings due to interference between the pixel beams which do not participate to the

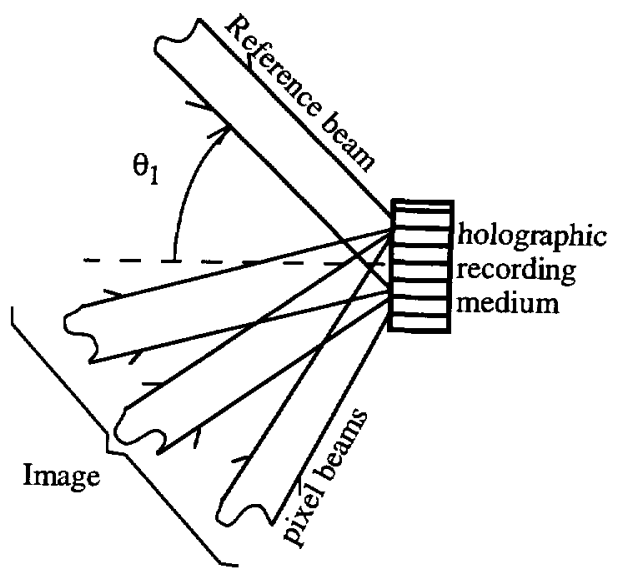

Fig. 1. - Recording angular multiplexing scheme chosen for calculation. The image beam is composed of $M$ plane pixel waves overlaping in the holographic material. 
information storage; second the gratings induced by the interferences between the pixel beams and the reference beam. For these $M$ last ones, the modulations of the interference pattern $m^{m, n}$ and grating wave vectors $\mathbf{k}_{\mathrm{g}}^{m, n}$ are :

$$
m^{m, n}=\frac{2\left|A_{\mathrm{ref}}^{n} A_{m}^{n}\right|}{\left(A_{\mathrm{ref}}^{n}\right)^{2}+\sum_{m=1}^{M}\left(A_{m}^{n}\right)^{2}} \quad \text { and } \quad \mathbf{k}_{\mathrm{g}}^{m, n}=\mathbf{k}_{\mathrm{ref}}^{n}-\mathbf{k}_{m}^{n}
$$

In order to slightly simplify our calculations, we assume that the image intensity $I_{\mathrm{rm}}=\sum_{m=1}^{M}\left(A_{m}^{n}\right)^{2}$ does not depend on the image we consider. This assumption is exact for pure phase modulation. It could also be a good approximation if pixel number $M$ is large enough. Equation (3a) thus simplifies to:

$$
m^{m, n}=\frac{2\left|A_{\mathrm{ref}}^{n} A_{m}^{n}\right|}{I_{\mathrm{ref}}+I_{\mathrm{lm}}} \quad \text { and } \quad \mathbf{k}_{\mathrm{g}}^{m, n}=\mathbf{k}_{\mathrm{ref}}^{n}-\mathbf{k}_{m}^{n}
$$

with $I_{\text {ref }}$ the reference beam intensity.

To determine the signal to noise ratio, we first need to find the relation between the modulation of the interference pattern and the amplitude of the refractive index (or absorption) modulation of the stored holograms. If only one hologram resulting from the interference of two uniform plane waves (i.e. one reference beam and a single pixel beam) of equal intensities is recorded until material saturation, then the maximum index modulation $\delta n_{\max }$ is reached. The material refractive index is thus :

$$
n(\mathbf{r})=n_{0}+\delta n_{\max } \sin \left(\mathbf{k}_{\mathbf{g}} \mathbf{r}\right)
$$

with $n_{0}$ the material refractive index when no grating is recorded.

Similarly, if only one hologram of a complex image is stored at saturation, then the index modulation $\delta n^{m, 1}$, corresponding to the grating with wave vector $\mathbf{k}_{\mathrm{g}}^{m .1}$, is equal to :

$$
\delta n^{m, 1}=m^{m, 1} \delta n_{\max } .
$$

If several images are sequentially recorded in the same material volume, then the index modulations are reduced. For photorefractive crystals, if the recording and erasing times are equal, then the index modulations $\delta n^{m, n}$ are inversely proportional to the number $N$ of superposed images :

$$
\delta n^{m, n}=m_{\mathrm{g}}^{m, n} \delta n_{\max } \quad \text { with } \quad m_{\mathrm{g}}^{m, n}=m^{m, n} / N
$$

$m_{\mathrm{g}}^{m, n}$ is the modulation ratio of the recorded grating.

This expression simply shows that the finite amount of electric charges which are used to record information is shared between the $N$ images. For color center materials, in which the bleaching of absorption $\alpha$ is proportional to the recording intensity, one obtains a similar expression. With obvious notations we get:

$$
\delta \alpha^{m, n}=m_{\mathrm{g}}^{m, n} \delta \alpha_{\max } .
$$

From equations (6) or (7), one sees that to maximize the retrieved signal (and thus to increase the signal to noise ratio), the modulations $m_{\mathrm{g}}^{m, n}$ of the stored patterns have to be maximized. 
This optimization depends on the type of data which is used. We will consider two types of data either with pure phase pixels or pure amplitude pixels.

3.1.1 Data encoded on the phase of the pixel beams. - In that case, the amplitude of all pixel beams is the same and equals to $A_{\mathrm{plx}}$. The modulations are maximum when $A_{\mathrm{plx}}=A_{\mathrm{ref}} / \sqrt{M}$ and we get :

$$
m_{\mathrm{g}}^{m, n}=1 /(N \sqrt{M})
$$

3.1.2 Data encoded on the amplitude of the pixel beams. - We now consider the case where each pixel intensity varies between two values, $I_{\mathrm{pix}}=I_{0} \neq 0$ and $I_{\mathrm{plx}}=0$. The average pixel intensity is $I_{\mathrm{pix}}^{\text {average }}=I_{0} / r$. The modulations for the pixels, with the largest intensity values $I_{0}$, are now maximum when $I_{0}=I_{\text {ref }} r / M$. We find :

$$
0 \leqslant m_{\mathrm{g}}^{m, n} \leqslant \sqrt{r} /(N \sqrt{M}) .
$$

Because it is difficult to perform phase detections (homodyne detections) on images, from now we only consider intensity detections. Furthermore, we restrict our analysis to amplitude data so that equation (9) must be used.

First we will quickly derive the expressions for signal and noise for absorption holograms in order to demonstrate the changes that must be performed on the ultimate capacity expressed by* equation (1). Then we will examine more deeply the case of index holograms recorded in photorefractive crystals.

3.2 FUNDAMENTAL SIGNAL TO NOISE RATIO AND MAXIMUM CAPACITY OF ANGULARLY MULTIPLEXED AMPLITUDE HOLOGRAMS. - We follow the analysis performed by van Heerden. The amplitude $a_{\text {read }}^{m, n}$ diffracted in the direction of a signal pixel beam is a random variable governed by a normal law. Its standard deviation (noise) is due to the random distribution of color centers and its mathematical expectation is proportional to the modulation ratio of the recorded grating. We thus have :

$$
E\left[a_{\text {read }}^{m, n}\right]=m_{\mathrm{g}}^{m, n}\left(N_{\mathrm{cc}} \mathrm{Vol}\right) \text { and } \sigma\left[a_{\mathrm{read}}^{m, n}\right] \approx \sqrt{N_{\mathrm{cc}} \mathrm{Vol}}
$$

On each pixel, one may store several bits encoded on amplitude levels. For instance if one wants to retrieve the data with an error rate of about $30 \%$, then one must fulfill the following equality :

$$
E\left[a_{\text {read }}^{m, n}(g-1)\right]+\sigma\left[a_{\text {read }}^{m, n}(g-1)\right]=E\left[a_{\text {read }}^{m, n}(g)\right]-\sigma\left[a_{\text {read }}^{m, n}(g)\right]
$$

where integer $g$ stands for the level number.

From equations (11) and (10) we find that two adjacent levels must be spaced by:

$$
E\left[a_{\text {read }}^{m, n}(g)\right]-E\left[a_{\text {read }}^{m, n}(g-1)\right] \approx 2 \sqrt{N_{\text {cc }} \text { Vol }} .
$$

Thus taking into account equation (9) and because we have $G+1$ levels ( $G$ positive levels plus zero), memory capacity $c=M N$ and integer $G$ are related by :

$$
\sqrt{N_{c c} \mathrm{Vol}} \sqrt{\frac{r}{M N^{2}}} \approx 2 G .
$$


One should remark that for intensity detection, the levels are not equally spaced and the noise depends on the level. The mathematical expectation of the detected intensity $I_{\text {read }}^{m, n}$ is indeed :

$$
E\left[I_{\text {read }}^{m, n}\right]=\left(E\left[a_{\text {read }}^{m, n}\right]\right)^{2}+\left(\sigma\left[a_{\text {read }}^{m, n}\right]\right)^{2}
$$

while its variance is :

$$
\left(\sigma\left[I_{\text {read }}^{m, n}\right]\right)^{2}=2\left(\sigma\left[a_{\text {read }}^{m, n}\right]\right)^{4}+4\left(\sigma\left[a_{\text {read }}^{m, n}\right]\right)^{2}\left(E\left[a_{\text {read }}^{m, n}\right]\right)^{2} .
$$

Equation (13) shows that for a given error rate and a given volume, capacity $c=M N$ is inversely proportional to the number $N$ of stored holograms. It is worth having few images with a large number of pixels rather than the opposite [27].

For instance, if one wants to record binary images $(G+1=2)$ with equal probabilities for the pixels to be on or off $(r=2)$, if $N_{\mathrm{cc}}=10^{15} \mathrm{~cm}^{-3}$ and $\mathrm{Vol}=5 \times 5 \times 5 \mathrm{~mm}^{3}$, for realistic values of pixel number $M=10^{6}$ pixels we find from equation (13) $N=8 \times 10^{3}$ images leading to a total capacity of $M \cdot N=8 \times 10^{9}$ pixels. This value is further reduced if gray level images are stored. Such values for the capacity are much lower than the ones derived from formula (1). The main reason for this discrepancy is that formula (1) can be applied for information recorded in a single exposition only while holographic data storage requires multiple exposures.

\section{Photorefractive holograms.}

4.1 AVAILABLE PHOTOREFRACTIVE MATERIALS FOR HOLOGRAPHIC STORAGE. - Many crystals present a photorefractive effect [10]. However, for photorefractive memories large photoinduced refractive index variations and long term storage in the dark are required. Only a few materials have these characteristics.

4.1.1 $\mathrm{Sr}_{(1-1)} \mathrm{Ba}_{1} \mathrm{Nb}_{2} \mathrm{O}_{6}$. - SBN seems to be the most promising crystal [28]. It has a large $r_{333}$ electrooptic coefficient which can be reached without a special crystal cut. The phase transition (that can be tuned by doping) is far away from the room temperature. It is also subject to electric or temperature fixing.

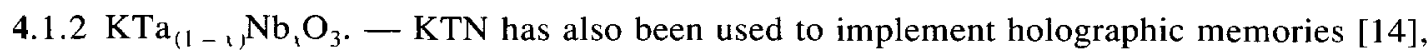
however nearly no datum has been published on this crystal.

4.1.3 $\mathrm{LiNbO}_{3}$. - Iron doped lithium niobate is the most commonly used material for demonstrations. The largest index modulations photoinduced in a photorefractive crystal were obtained in lithium niobate using the photovoltaic effect. Furthermore it is available in very large dimensions with high optical quality. For instance a $3 \mathrm{~cm}^{3}$ sample was used to record the 5000 holograms in [6]. Because of its strong mechanical properties, lithium niobate was also employed to construct holographic disks $[29,30]$.

4.1.4 $\mathrm{BaTiO}_{3}$. - To achieve large diffraction efficiencies in lithium niobate crystals one must use the photovoltaic effect. First this imposes the use of short wavelengths (usually $488 \mathrm{~nm}$ ) and second the photovoltaic effect may lead to the distortion of the phase of the image beam wavefront. For these reasons, $\mathrm{BaTiO}_{3}$ crystals are sometimes preferred $[9,27]$. However they also present some other drawbacks. Crystal are not available in large dimensions, a $5 \times 5 \times 5 \mathrm{~mm}^{3}$ sample is considered as a «big» sample. To reach the maximum index modulations, the sample must be cut at a certain angle of the crystallographic axis $\dot{c}$. This cut is difficult and it reduces the size of the sample. A phase transition exists around $13{ }^{\circ} \mathrm{C}$ so that the crystal must always be kept above this temperature. 
Because the ultimate capacity of photorefractive materials depends on the type of crystal we use, in the following we will base all our calculations on a $\mathrm{BaTiO}_{3}$ sample.

Figure 2 shows a plot of the maximum refractive index modulation $\delta n_{\mathrm{mdx}}$ that can be induced in such a sample. The sample is cobalt doped in order to increase the magnitude of the photorefractive effect [31]. The two interfering beams are extraordinary polarized and travel in a plane perpendicular to the crystallographic $\vec{b}$ axis. The modulation ratio of the interference pattern is one. $\theta_{1}$ and $\theta_{2}$ are the angles between the wave vectors and the $\hat{a}$ axis. The index modulation is symmetrical around $\theta_{1}=\theta_{2}$ for which $\delta n=0$. One sees that the index modulation strongly depends on the difference $\theta_{1}-\theta_{2}$ thus on the fringe spacing. This dependence is mainly due to the variation of the photoinduced electric field with the grating fringe spacing [10]. It also varies but more slowly with $\theta_{1}+\theta_{2}$, that is with the orientation of the grating planes. This variation arises from the variation of the electrooptic effect with the orientation of the electric field. These dependencies are valid for all photorefractive materials. Figure 2 demonstrates that a small part of the $\left(\theta_{1}, \theta_{2}\right)$ plane is permitted only if one wants a sizable refractive index modulation. Thus, the number of elementary storage cells will be much lower than the maximum $c \approx \mathrm{Vol} / \lambda^{3}$.

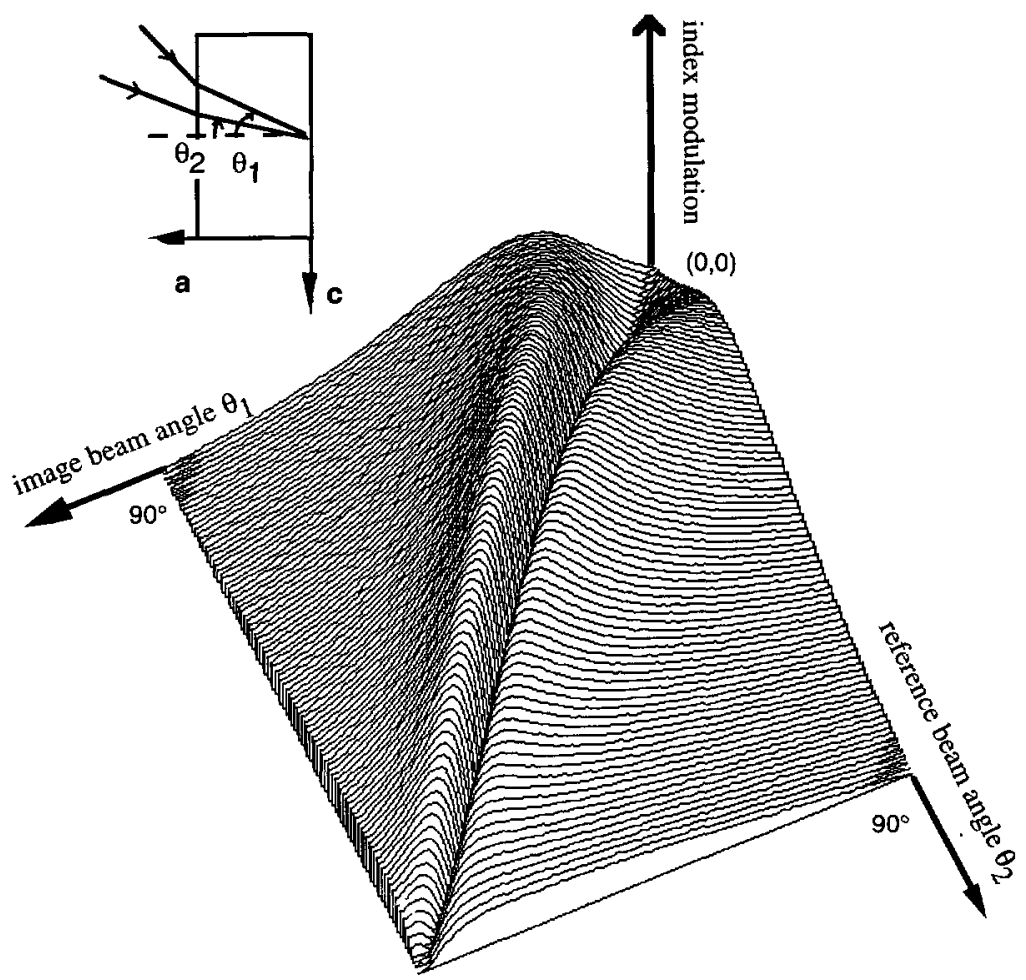

Fig. 2. - Absolute value of the photoinduced modulation of the refractive index in the $\mathrm{Co}: \mathrm{BaTiO}_{3}$ as a function of propagation directions $\theta_{1}, \theta_{2}$ of the 2 extraordinary polarized interfering beams; the trap density is $N_{\mathrm{A}}=4 \times 10^{22} \mathrm{~m}^{-3}$

This observed dependence of index modulation on $\theta_{1}$ and $\theta_{2}$ is general for all materials in which the photorefractive effect is driven by diffusion mechanisms. Different dependencies are 
found when the photorefractive effect is driven by an applied electric field or by the photovoltaic effect [10].

4.2 Fundamental SIGNAL TO NOISE RATIO OF PHOTOREFRACTIVE HOLOGRAMS. - We here present a simplified approach for the determination of the signal to noise ratio limitation due to finite number of photorefractive centers. A more detailed analysis is given in [32]. Because we are looking for limitations due to noise, we simplify the following analysis by considering small diffraction efficiencies only. We thus assume, first no beam depletion, and second that the scattered light is not amplified by photorefractive two wave mixing (beam fanning phenomenon) [10].

In the dark, the charges (ex. . electrons) are randomly distributed among the charge centers whose density is $N_{\mathrm{d}}$. When these centers are filled with an electron, they are electrically neutral. Some of them remain empty of electrons and present a positive charge. Their density is $N_{\mathrm{d}}^{+}$. Because the crystal is electrically neutral, there exist other negative charge centers whose density is $N_{\mathrm{A}}$. These centers, usually called «photorefractive trap centers », are inactive for the photorefractive effect. To assure electric neutrality, the average values of densities $N_{\mathrm{d}}^{+}$and $N_{\mathrm{A}}$ over the crystal volume are equal. The trap centers $N_{\mathrm{A}}$ are randomly distributed in the material. When no grating is recorded, the centers $N_{\mathrm{d}}^{+}$are also randomly distributed. The redistribution of electrons among the charge centers leads to a new distribution of charges and thus to the creation of a new electric field pattern. The resulting index modulation is proportional to the electric field amplitude. For a single electric field grating, whose modulation is $E_{1}$, Poisson's equation, links $E_{1}$ to the modulation amplitude $m N_{\mathrm{d}}^{+1}$ of centers $N_{\mathrm{d}}^{+}$.

$$
m N_{\mathrm{d}}^{+1}=\frac{\varepsilon k_{\mathrm{g}} m E_{1}}{e}
$$

with $k_{\mathrm{g}}$ the grating wave number, $\varepsilon$ the dielectric constant, $e$ the charge of the electron and $m$ the modulation ratio of the stored grating. If only one grating is recorded in the material, this modulation ratio is equal to the modulation ratio of the interference pattern. The index modulation is proportional to the electric field and thus to $N_{\mathrm{d}}^{+1}$. To compute $\delta n_{\max }$ one must thus find the maximum electric field that can be photoinduced. This field $E_{1}$ can be calculated from the theory of the photorefractive effect [10].

To compute the signal to noise ratio, we consider a single datum (i.e. one pixel of one image) written in the material by interfering two plane waves. A uniform charge grating modulated along the $x$ axis appears in the material. In steady state, the charge distribution is written as :

$$
N_{\mathrm{d}}^{+}=N_{\mathrm{A}}\left(1+m \frac{N_{\mathrm{d}}^{+1}}{N_{\mathrm{A}}} \sin \left(k_{\mathrm{g}} x\right)\right)
$$

Following a macroscopic approach, this represents the average distribution of charge density. For large $N_{\mathrm{d}}^{+}$, in a microscopic approach, the mathematical expectation equals this mean value. Thus the mathematical expectation of charge number $n(x) \mathrm{d} x$ (random variable) distributed in the slice of volume $h \ell \mathrm{d} x$ (thickness $\ell$, width $\mathrm{d} x$ ) is :

$$
E[n(x) \mathrm{d} x]=E[n(x)] \mathrm{d} x=N_{\mathrm{A}}\left(1+m \frac{N_{\mathrm{d}}^{+1}}{N_{\mathrm{A}}} \sin \left(k_{\mathrm{g}} x\right)\right) h \ell \mathrm{d} x
$$


Assuming that all charges are independent, the probability law for $n(x) \mathrm{d} x$ follows a Poisson's law giving a variance :

$$
\sigma^{2}[n(x) \mathrm{d} x]=E[n(x) \mathrm{d} x] .
$$

This equation is only an approximation in case where the charges are not independent. For instance, the Coulomb interaction is taken into account in [32].

The diffracted amplitude is proportional to the Fourier transform of $n(x)$. Again, for independent charges, one gets the mathematical expectation of the Fourier transform of $n(x)$ :

$$
E[\mathrm{FT}(n(x))]=E\left[\frac{2}{a} \int_{0}^{a} \sin \left(k_{\mathrm{g}}^{\prime} x\right) n(x) \mathrm{d} x\right]=m h \mathcal{R}_{\mathrm{d}}^{+1} \operatorname{sinc}\left[\left(k_{\mathrm{g}}-k_{\mathrm{g}}^{\prime}\right) a\right]
$$

$a$ being the crystal size along the $x$ direction and $k_{\mathrm{g}}^{\prime}$ the wave vector of the observed Fourier component.

Taking into account equation (19), its variance is :

$$
\sigma^{2}[F T(n(x))]=2 N_{\mathrm{A}} h \ell / a .
$$

This variance corresponds to the amplitude diffracted by the random distribution of charge centers $N_{\mathrm{d}}^{+}$. Actually, we must also take into account the amplitude diffracted by the charge centers $N_{\mathrm{A}}$. Thus the variance for the amplitude is :

$$
\sigma_{\mathrm{ampl}}^{2}=2 \sigma^{2}[\mathrm{FT}(n(x))] .
$$

Thus once again, taking into account equation (9), for an error rate of about $30 \%$, we find the following relation between capacity $c=M N$ and integer $G$ :

$$
2 G=\frac{E[\mathrm{FT}(n(x))]}{\sigma_{\text {ampl }}[\mathrm{FT}(n(x))]}=\sqrt{\frac{r}{M N^{2}}} \sqrt{\frac{\text { Vol }}{N_{\mathrm{A}}}} \frac{N_{\mathrm{d}}^{+1}}{2}
$$

in which we have considered signal detected in direction $k_{\mathrm{g}}=k_{\mathrm{g}}$.

Following the discussion of section 3 , the number of levels for amplitude detection is $G+1$.

The maximum signal, and thus the maximum capacity is obtained for the maximum value of $N_{\mathrm{d}}^{+1}$ At most we have $N_{\mathrm{d}}^{+1}=N_{\mathrm{A}} / 2$ [10]. From this value and from equation (23) one easily derives the ultimate capacity.

One should remember that the capacity expressed by (23) is overestimated because many other sources for noise are neglected.

4.3 CAPACITY LIMITED By GEOMETRICAL CONSTRAINTS. - The maximum number of elementary storage cells that can be defined in any holographic medium was precisely calculated in reference [33]. The authors show that expression $c \approx \mathrm{Vol} / \lambda^{3}$ underestimates the total number of cells. Their results also apply for other holographic storage materials such as color centers materials. Because of the dependence of the photorefractive effect on the fringe spacing and on the orientation of the grating wave vector, only a small number of these holograms can be recorded. Hereafter, we will derive a crude estimation for the number of allowable cells in the case of a $\mathrm{BaTiO}_{3}$ sample.

4.3.1 Pixel number per image in photorefractive crystals. - The number of pixels in a stored image is ultimately limited by diffraction and thus by the crystal aperture. However because of 
the dependence of the photorefractive grating strength on the fringe spacing and on its orientation, the image beam must be contained in a solid angle $\Delta \theta_{2} \times \Delta \Phi_{2}$ so that the diffraction efficiency remains high for all image components. This condition is also required to keep the signal to noise ratios at their maximum. With a crystal with square entrance surface $h \times h$, and because $\Delta \theta_{2} \approx \Delta \Phi_{2}$, we get :

$$
M=\left(\Delta \theta_{2} h / \lambda\right)^{2}
$$

For a common $\mathrm{BaTiO}_{3}$ crystal of entrance surface $5 \times 5 \mathrm{~mm}^{2}$, each image may have up to $M \approx 10^{7}$ pixels if the diffraction efficiency is kept around its maximum [34]. Of course, this number can be increased but at the expense of diffraction efficiencies and of signal to noise ratios. Such high resolution images have been stored in photorefractive crystals in two wave mixing experiments (single dynamic image amplification) only. In these experiments [35] the input image is a projector slide and does not have to be changed. However for optical memories an input spatial light modulator is required to impress and to change the data on the image beam. Thus in most experimental demonstrations of photorefractive memory, the ultimate resolution is not reached but is limited by the number of pixels of the input spatial light modulator.

The limitation imposed by the limited surface of the holographic medium appears in some cases. For instance, photorefractive fibers were used to record images. The small entrance surface (e.g. $200 \mu \mathrm{m}$ in diameter for Ref. [36]) strongly limits the image resolution.

4.3.2 Number of images stored in a photorefractive crystal. - As explained above, to avoid cross talk between reconstructed images, we take advantage of the Bragg selectivity. The reference beam wave vectors are in the same incidence plane and the angular spacing $A_{\theta}$ between the reference beams is chosen to be larger than the Bragg angular selectivity $\Delta \theta_{\mathrm{B}}$ to avoid crosstalk. Because the diffraction éfficiency of photorefractive gratings strongly depends on their orientations and fringe spacings, the allowable angular aperture for the reference beams $\Delta \theta_{1}$ must be restricted to get uniform diffraction efficiencies. This aperture depends on the photorefractive parameters. For transmission holograms, the maximum number of images $N$ that can be stored in the crystal is about :

$$
N \approx \frac{\Delta \theta_{1}}{A_{\theta}} \leqslant \frac{\Delta \theta_{1}}{\Delta \theta_{\mathrm{B}}} \approx \Delta \theta_{1} \frac{\text { crystal thickness }}{\text { fringe spacing }}
$$

For example, with a crystal thickness of $5 \mathrm{~mm}$, an average fringe spacing of $3 \mu \mathrm{m}$ and an allowable angular aperture $\Delta \theta_{1} \approx 10^{\circ}$ we find $N \leqslant 290$ superposed holograms. This number of images can be slightly increased by using sampling grids as described in [6]. A more detailed analysis for the image capacity is conducted in reference [33].

\section{Data rate-capacity trade off.}

We do not only want large capacities but we also desire high data rates. Of course, the power $P$ of the readout beam is limited. The diffracted signal must thus be integrated during a long time enough to get a sufficient photon number per pixel to reconstruct the image [37]. Because the readout time $\tau$ is limited, and because the diffraction efficiency decreases when the number of images increases, we have here a strong limitation on the memory capacity. If $n_{\mathrm{b}}$ is the photon number required for deciding the pixel value, the data rate for images is expressed as :

$$
n_{\mathrm{b}}=\left(m_{\mathrm{g}}^{m, n}\right)^{2} P \tau D_{\max } n_{0} \text { with } D_{\max }=\left(\pi \delta n_{\max } P / \lambda_{0}\right)^{2}
$$


$\lambda_{0}$ being the optical wavelength in vacuum, $n_{0}$ the photon number in 1 Joule $\left(n_{0}=\lambda_{0} / h c\right.$, $h$ Planck's constant and $c$ light velocity in vacuum).

As explained in paragraph 4.2 , equation (26) has been written considering small diffraction efficiencies $\left(\sin ^{2}[x] \approx x^{2}\right)$.

The detection error rate depends on signal $n_{\mathrm{b}}$, on detector noise and on shot noise $\sqrt{n_{\mathrm{b}}}$.

\section{IMAGE MEMORIES.}

5.1.1 Binary images. - If we deal with binary images, we have to detect the presence or the absence of a signal. The main source of noise is thus the detector noise. We may choose a reasonable value $n_{\mathrm{b}}=10^{3}$ photons. Thus for a reading power of $10 \mathrm{~mW}$ at $532 \mathrm{~nm}$, a diffraction efficiency per hologram $D_{\text {max }} / N^{2}$ of $0.1 \%$ (which is readily achievable with $N=256$ stored images) and coefficient $r=2$, we get a maximum information rate of $M / \tau \approx 5 \times 10^{10} \mathrm{~s}^{-1}$ Consequently, for images of $M=256 \times 256$ pixels, i.e. a total capacity of $2 \times 10^{7}$ bits, the minimum image reading time becomes $\tau \approx 2 \mu \mathrm{s}$.

5.1.2 Gray level images. - If images with many gray levels, $G+1 \gg 2$, are stored, the shot noise becomes the most important source of noise. The intensity signal is a random variable with mathematical expectation $E\left[I_{\text {read }}^{m, n}(g)\right]=n_{\mathrm{b}}(g)$ and standard deviation $\sigma\left[I_{\text {read }}^{m, n}(g)\right]=$ $\sqrt{n_{\mathrm{b}}(g)}$. If all other noise sources are neglected, to record images with gray levels, and to retrieve them with an error rate of about $30 \%$, we must have :

$$
E\left[I_{\text {read }}^{m, n}(g)\right]-\sigma\left[I_{\text {read }}^{m, n}(g)\right]=E\left[I_{\text {read }}^{m, n}(g-1)\right]+\sigma\left[I_{\text {read }}^{m, n}(g-1)\right] .
$$

For $G+1$ equally spaced gray levels, we get the following expression from equations (9) and (27):

$$
\frac{\sqrt{r P \tau D_{\max } n_{0}}}{G N \sqrt{M}} \geqslant 2 .
$$

For example, with the same parameters as previously, except $G+1=128$ gray levels, we now find $M / \tau \approx 8 \times 10^{8} \mathrm{~s}^{-1}$ The minimum image reading time now rises to $\tau \approx 80 \mu \mathrm{s}$.

5.2 FILTERS MEMORIES FOR CORRELATORS. - Once again, we want to point out that the above calculation is valid for incoherent detection only. If coherent detection is performed the results are totally different. For example, if the images are used to feed a Vander Lugt correlator, then the images stored in the memory are not detected. the detection is performed in the correlation plane and the amount of photons which are needed to localize the correlation peak depends only on the signal to noise ratio of this peak. As the limit, if the stored images correspond to orthogonal modes of the electromagnetic fields and if the Vander Lugt filter corresponds to one of these modes then only one photon is required to determine the filter mode. The memory capacity is thus much larger in that case.

\section{An example of accessible storage capacity.}

We will now gather all our results to study the storage capacity of a $\mathrm{Co}: \mathrm{BaTiO}_{3}$ sample $\left(N_{\mathrm{A}}=4 \times 10^{22} \mathrm{~m}^{-3}\right)$. We choose to realize an image memory with $G+1=64$ gray levels and $r=2$. The size of the recording volume is $5 \times 5 \times 5 \mathrm{~mm}^{3}$. The gratings are recorded and 
retrieved with a $P=10 \mathrm{~mW}$ laser beam at $\lambda_{0}=532 \mathrm{~nm}(\lambda=220 \mathrm{~nm}$ in the crystal $)$. For such a sample, at the top of the refractive index modulation surface in figure 2 , we measure $\delta n_{\max }^{\text {top }}=3.5 \times 10^{-4}$ We choose to work around the top of the modulation index surface. We will limit the variation of the diffraction efficiency of the different images to a factor of two. It gives $2.5 \times 10^{-4} \leqslant \delta n_{\max } \leqslant 3.5 \times 10^{-4}$ which approximately corresponds to $N_{\mathrm{A}} /(2 \sqrt{2}) \leqslant$ $N_{\mathrm{d}}^{+1} \leqslant N_{\mathrm{A}} / 2$. The number of pixels in the image beam is also limited by the input intensity image modulator or slide to $M=10^{7}$ pixels. From these data and from equations (23), (24), (25) and (28) we get the following limits.

\subsection{CAPACITY LIMITED BY NOISE :}

$$
M N \leqslant \frac{r N_{\mathrm{A}} \text { Vol }}{128 G^{2} N} \approx \frac{2 \times 10^{10}}{N}
$$

6.2 PIXEL AND IMAGE NUMBERS LIMITED BY GEOMETRICAL CONSTRAINTS. - In principle, to determine the maximum number of image and pixels, we must cut the 3D surface in figure 2 by the plane $\delta n_{\max }=\delta n_{\max }^{\text {top }} / 2$. The intersection of the two surfaces defines the largest angular apertures and thus $M$ and $N$. For simplicity we choose identical values for the angular apertures. However, a different choice could also be made. We get $\Delta \theta_{2} \approx 10^{\circ}, \Delta \theta_{1} \approx 10^{\circ}$ so that :

$$
\begin{aligned}
& M \leqslant 1.5 \times 10^{7} \text { pixels } \\
& N \leqslant 290 \text { images }
\end{aligned}
$$

\subsection{DATA RATE-CAPACITY TRADE OFF :}

$$
\frac{M N}{\tau} \leqslant \frac{r P D_{\max } n_{0}}{4 G^{2} N}=\frac{2 \times 10^{14}}{N} s^{-1}
$$

These equations (29) to (32) define a volume in the coordinate system $(M, N, \tau)$ which is drawn in figure 3.

Of course, this volume could by modified by taking a different choice for the parameters but it gives a good representation of what can be achieved with presently available photorefractive materials. Indeed, the use of the same equations (23), (24), (25) and (28) leads to different accessible capacity volumes for other photorefractive samples and other configurations.

\section{Conclusion.}

We have pointed out the drastic capacity reduction of the multiple exposure holographic storage compared to the single exposure storage previously described by van Heerden. In case of photorefractive holograms, and taking $\mathrm{BaTiO}_{3}$ crystals as examples, we specified the accessible storage capacity versus various parameters. One may feel disappointed by the relatively low storage capacity of these materials (about $10^{9}$ gray level pixels in Fig. 3). However, one must keep in mind that these storage capacities are readily accessible with today technologies. This point plus the parallelism inherent to these memories open very attractive perspectives for optical data processing devices, such as correlators, neural networks and even digital computing units [27]. 


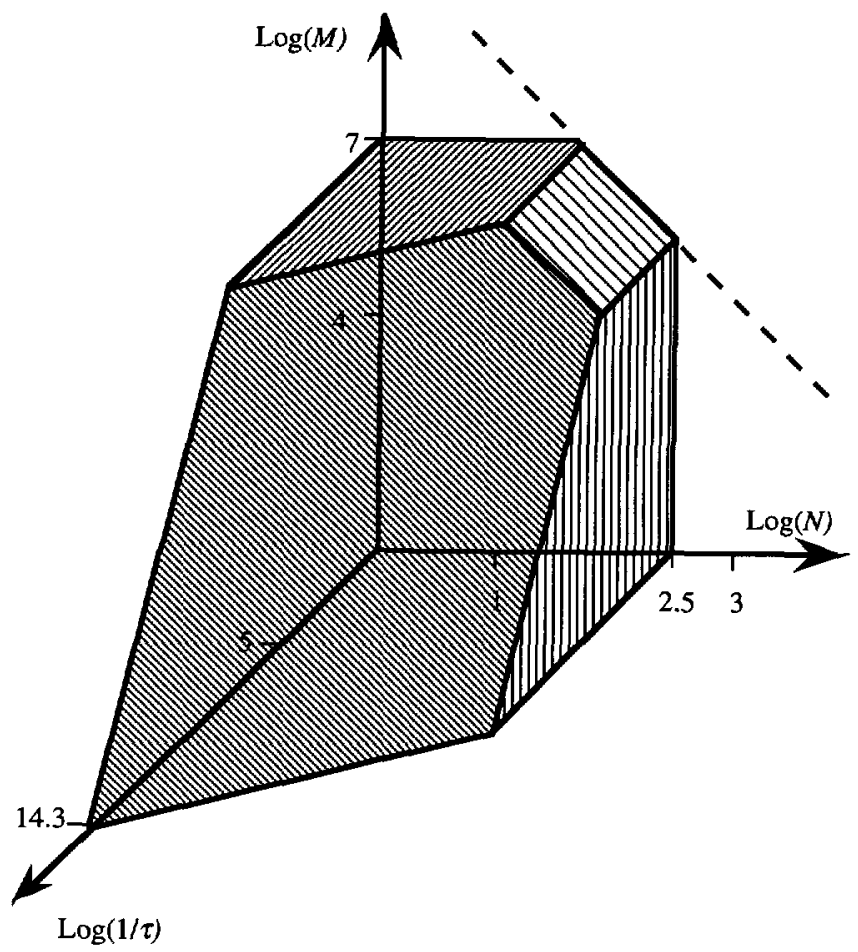

Fig. 3. - Typical accessible (number of image, number of pixel, readout time) volume for a photorefractive crystal. The dashed line corresponds to the intersect of the plane $(\log (M)$, $\log (N)$ ) with the limit imposed by the noise due to the random distribution of electric charges.

\section{References}

[1] d'Auria L., Huignard J. P., Spitz E., Holographic Read-Write Memory and Capacity Enhancement by 3-D Storage, IEEE Trans. Magn. 9 (1973) 83-94.

[2] d'Auria L., Huignard J. P., Slezak C., Spitz E., Experimental Holographic Read-Write Memory Using 3-D Storage, Appl. Opt. 13 (1974) 808-818.

[3] Staebler D. L., Burke W. J., Phillips W., Amodei J. J., Multiple storage and erasure of fixed holograms in Fe-doped LiNbO 3 , Appl. Phys. Lett. 26 (1975) 182-184.

[4] Kurz H., Photorefractive recording dynamics and multiple storage of volume holograms in photorefractive $\mathrm{L}_{1} \mathrm{NbO}_{3}$, Optica Acta 24 (1977) 463-473.

[5] Mok F. H., Tackitt M. C., Stoll H. M., Storage of 500 high-resolution holograms in a $\mathrm{LiNbO}_{3}$ crystal, Opt. Lett. 16 (1991) 605-607.

[6] Mok F. H., Angle-multiplexed storage of 5000 holograms in lithium niobate. Opt. Lett. 18 (1993) 915-917.

[7] Taketomi Y., Ford J. E., Sasaki H., Ma J., Fainman Y., Lee S. H., Incremental recording for photorefractive hologram multiplexing, Opt. Lett. 16 (1991) 1774-1776.

[8] Denz C., Pauliat G., Roosen G., Tschudi T., Volume hologram multiplexing using a deterministic phase encoding method, Opt. Commun. 85 (1991) 171-176.

[9] Pauliat G., Roosen G., New advances in photorefractive holographic memories, Int. Opt. Computing, Special issue on «Photorefractive materials » Issue 32 (1991) 271-291.

[10] Photorefractive Materials and Their Applications I, P. Günter, J. P. Huignard Eds. 61 (Springer Verlag Berlın, 1988). 
[11] Amodei J. J., Staebler D. L., Holographic pattern fixing in electro-optic crystals, Appl. Phys. lett. 18 (1971) 540-542.

[12] Micheron F., Bismuth G., Electrical control of fixation and erasure of holographic patterns in ferroelectric materials, Appl. Phys. Lett. 20 (1972) 79-81.

[13] Micheron F., Bismuth G.. Field and time thresholds for the electrical fixation of holograms recorded in $\left(\mathrm{Sr}_{075} \mathrm{Ba}_{025}\right) \mathrm{Nb}_{2} \mathrm{O}_{6}$ crystals, Appl. Phys. lett. 23 (1973) 71-72.

[14] Leyva V., Agranat A., Yariv A.. Fixing of a photorefractive grating in $\left.\mathrm{KTa}_{(1-\mathrm{x}}\right) \mathrm{Nb}_{(\mathrm{x})} \mathrm{O}_{3}$ by cooling through the ferroelectric phase transition. Opt. Lett. 16 (1991) 554-556.

[15] McCahon S. W., Rytz D., Valley G. C., Klein M. B., Wechsler B. A., Hologram fixing in $\mathrm{Bi}_{12} \mathrm{Ti}_{020}$ using heating and an AC electric field, Appl. Opt. 28 (1989) 1967-1969.

[16] Redfield S., Hesselink L., Enhanced nondestructive holographic readout in strontium barium niobate, Opt. Lett. 13 (1988) 880-882.

[17] Kewitsch A., Segev M., Yariv A., Neurgaonkar R., Selective page-addressable fixing of volume holograms in $\mathrm{Sr}_{075} \mathrm{Ba}_{0}{ }_{25} \mathrm{Nb}_{2} \mathrm{O}_{6}$ crystals, Opt. Lett. 18 (1993) 1262-1264.

[18] Von der Linde D., Glass A. M., Rodgers K. F.. Multiphoton photorefractive processes for optical storage in $\mathrm{LiNbO}_{3}$, Appl. Phys. 25 (1974) 155-157.

[19] Külich H. C., Reconstructing volume holograms without image field losses, Appl. Opt. 30 (1991) 2850-2857.

[20] Brady D., Hsu K., Psaltis D., Periodically refreshed multiply exposed photorefractive holograms, Opt. Lett 15 (1990) 817-819.

[21] Qiao Y.. Psaltis D., Gu C., Hong J., Yeh P., Neurgaonkar R. R., Phase-locked sustainment of photorefractive holograms using phase conjugation, J. Appl. Phys. 17 (1991) 4646-4648.

[22] Qiao Y., Psaltis D.. Sampled dynamic holographic memory, Opt. Lett. 17 (1992) 1376-1378.

[23] Boj S., Pauliat G., Roosen G., Dynamic holographic memory showing read-out, refreshing and updating capabilities. Opt. Lett. 17 (1992) 438-440.

[24] Kogelnik H., Coupled wave theory for thick hologram gratings, Bell Syst. Tech. J. 48 (1969) 29092947.

[25] van Heerden P. J., Theory of Optical Information Storage in Solids, Appl. Opt. 2 (1963) 393-400.

[26] Burr G. W., Psaltis D., Curtis K., « Comparison of wavelength and angle multiplexed holographic memories », proceeding of the Optical Computing topical meeting, paper OWB4-1 (1993) pp. 87-90.

[27] Alves C., Aing P., Paulıat G., Roosen G., Photorefractive memories for optical processing and computing, Crittcal Review's SPIE CR48 (1993) 221-228.

[28] Ewbank M. D., Neurgaonkar R. R., Cory W. K., Photorefractive properties of strontium-barium niobate, J. Appl. Phys 62 (1987) 374-380.

[29] Psaltis D., Neifeld M. A.. Yamamura A., Image correlators using optical memory disks, Opt. Lett. 14 (1989) 429-431.

[30] Yamamura A. A., Neifeld M. A., Kobayashi S., Psaltis D., Optical disk based artificial neural systems, Opt. Computing Processing 1 (1991) 3-12.

[31] Rytz D., Wechsler B. A., Garrett M. H., Nelson C. C., Schwartz R. N., Photorefractive properties of $\mathrm{BaTiO}_{3}: \mathrm{Co}$, JOSA B 7 (1990) 2245-2254.

[32] Gu C., Yeh P., Scatterıng due to randomly distributed charge particles in photorefractive crystals, Opt. Lett. 16 (1991) 1572-1574.

[33] Brady D., Psaltis D., Information capacity of 3-D holographic data storage, Opt. Q. Electron. 25 (1993) 597-610.

[34] Pauliat G. and Roosen G., Interconnects with optically thick elements, chap 13 in Perspective for parallel Optical Interconnects Ph. Lalanne, P. Chavel Eds., (Basic Research Series Springer Verlag, 1993).

[35] Fainman Y., Klancnik E., Lee S. H., Optimal coherent image amplification by two-wave coupling in photorefractive $\mathrm{BaTiO}_{3}$, Opt. Eng. 25 (1986) 228-234.

[36] Yoshinaga H., Kitayama K. I., Oguri H., Holographic image storage in iron doped lithium niobate fibers. Appl. Phys. Lett. 56 (1990) 1728-1730.

[37] Bløtekjaer K., Limitations on holographic storage capacity of photochromic and photorefractive media, Appl. Opt. 18 (1979) 57-67. 